\title{
Head of bed elevation in pediatric patients with severe traumatic brain injury
}

\author{
Shih-Shan Lang, MD, ${ }^{1,2}$ Amber Valeri, DO, ${ }^{3}$ Bingqing Zhang, MPH, ${ }^{4}$ Phillip B. Storm, MD, ${ }^{1,2}$ \\ Gregory G. Heuer, MD, PhD, ${ }^{1,2}$ Lauren Leavesley, MD, ${ }^{5}$ Richard Bellah, MD, ${ }^{6}$ Chong Tae Kim, MD, ${ }^{7}$ \\ Heather Griffis, PhD, ${ }^{4}$ Todd J. Kilbaugh, MD, ${ }^{5}$ and Jimmy W. Huh, MD ${ }^{5}$
}

\begin{abstract}
'Division of Neurosurgery, Children's Hospital of Philadelphia, Department of Neurosurgery, University of Pennsylvania, Perelman School of Medicine; ${ }^{2}$ Center for Data Driven Discovery in Biomedicine, Children's Hospital of Philadelphia; ${ }^{3}$ Department of Neurosurgery, Philadelphia College of Osteopathic Medicine; ${ }^{4}$ Healthcare Analytics Unit, Department of Biomedical and Health Informatics, Children's Hospital of Philadelphia; ${ }^{5}$ Department of Anesthesiology and Critical Care Medicine, Children's Hospital of Philadelphia, University of Pennsylvania, Perelman School of Medicine; ${ }^{\circ}$ Department of Radiology and Pediatrics, Children's Hospital of Philadelphia, University of Pennsylvania, Perelman School of Medicine; and 'Department of Physical Medicine and Rehabilitation and Pediatrics, Children's Hospital of Philadelphia, University of Pennsylvania, Perelman School of Medicine, Philadelphia, Pennsylvania
\end{abstract}

\begin{abstract}
OBJECTIVE Head of bed (HOB) elevation to $30^{\circ}$ after severe traumatic brain injury (TBI) has become standard positioning across all age groups. This maneuver is thought to minimize the risk of elevated ICP in the hopes of decreasing cerebral blood and fluid volume and increasing cerebral venous outflow with improvement in jugular venous drainage. However, HOB elevation is based on adult population data due to a current paucity of pediatric TBI studies regarding HOB management. In this prospective study of pediatric patients with severe TBI, the authors investigated the role of different head positions on intracranial pressure (ICP), cerebral perfusion pressure (CPP), and cerebral venous outflow through the internal jugular veins (IJVs) on postinjury days 2 and 3 because these time periods are considered the peak risk for intracranial hypertension.
\end{abstract}

METHODS Patients younger than 18 years with a Glasgow Coma Scale score $\leq 8$ after severe TBI were prospectively recruited at a single quaternary pediatric intensive care unit. All patients had an ICP monitor placed, and no other neurosurgical procedure was performed. On the 2 nd and 3 rd days postinjury, the degree of $\mathrm{HOB}$ elevation was varied between $0^{\circ}$ (head-flat or horizontal), $10^{\circ}, 20^{\circ}, 30^{\circ}, 40^{\circ}$, and $50^{\circ}$ while ICP, CPP, and bilateral IJV blood flows were recorded.

RESULTS Eighteen pediatric patients with severe TBI were analyzed. On each postinjury day, 13 of the 18 patients had at least 1 optimal HOB position (the position that simultaneously demonstrated the lowest ICP and the highest CPP). Six patients on each postinjury day had $30^{\circ}$ as the optimal HOB position, with only 2 being the same patient on both postinjury days. On postinjury day 2, 3 patients had more than 1 optimal HOB position, while 5 patients did not have an optimal position. On postinjury day 3, 2 patients had more than 1 optimal HOB position while 5 patients did not have an optimal position. Interestingly, $0^{\circ}$ (head-flat or horizontal) was the optimal HOB position in 2 patients on postinjury day 2 and 3 patients on postinjury day 3 . The optimal HOB position demonstrated lower right IJV blood flow than a nonoptimal position on both postinjury days $2(p=0.0023)$ and $3(p=0.0033)$. There was no significant difference between optimal and nonoptimal HOB positions in the left IJV blood flow.

CONCLUSIONS In pediatric patients with severe TBI, the authors demonstrated that the optimal HOB position (which decreases ICP and improves CPP) is not always at $30^{\circ}$. Instead, the optimal HOB should be individualized for each pediatric TBI patient on a daily basis.

https://thejns.org/doi/abs/10.3171/2020.4.PEDS20102

KEYWORDS head of bed elevation; cerebral perfusion pressure; intracranial pressure; pediatric; traumatic brain injury; trauma; neuromonitoring; neurocritical care

\footnotetext{
ABBREVIATIONS ABP = arterial blood pressure; $\mathrm{CBF}=$ cerebral blood flow; $\mathrm{CPP}=$ cerebral perfusion pressure; $\mathrm{GCS}=$ Glasgow Coma Scale; GOS-E = Glasgow Outcome Scale-Extended; $\mathrm{HOB}=$ head of bed; ICP = intracranial pressure; IJV = internal jugular vein; $\mathrm{MAP}=$ mean arterial pressure; $\mathrm{TBI}=$ traumatic brain injury; US = ultrasound.
}

SUBMITTED February 10, 2020. ACCEPTED April 27, 2020.

INCLUDE WHEN CITING Published online July 17, 2020; DOI: 10.3171/2020.4.PEDS20102. 
$\mathrm{P}$ EDIATRIC traumatic brain injury (TBI) remains one of the leading causes of long-term disability and mortality worldwide. ${ }^{1-4}$ The cornerstone of acute neurocritical care management in severe pediatric TBI is to prevent or treat elevated intracranial pressure (ICP) and low cerebral perfusion pressure (CPP). ${ }^{5}$ Elevation of the head of the bed (HOB) to $30^{\circ}$ in a neutral position has become the standard management for the treatment of ICP in the hopes of decreasing cerebral blood and fluid volume and increasing cerebral venous outflow with improvement in jugular venous drainage.$^{6-10}$ Due to a paucity of prospective studies or randomized control trials regarding management of severe TBI in the pediatric population, most management strategies have been extrapolated from adult studies. However, HOB positioning studies in adults with severe TBI have demonstrated conflicting results. ${ }^{11-20}$ To date, there has only been one study on the effects of HOB positioning on ICP and CPP in the pediatric population following severe TBI. An analysis of 8 pediatric patients who sustained severe TBI demonstrated that in most but not all cases higher HOB elevation resulted in lower ICP without affecting CPP. ${ }^{21}$

Previous studies have analyzed different HOB positions during only a single day following severe TBI. Furthermore, no studies have analyzed the association of HOB position and cerebral venous outflow via ultrasonography of the bilateral internal jugular veins (IJVs). In this study, we evaluated the role of different HOB positions on ICP and CPP on 2 consecutive days, postinjury days 2 and 3 , because these time periods are considered the peak risk for intracranial hypertension following severe TBI. Our hypothesis was that the optimal HOB position-the angle of head elevation that minimizes ICP and promotes the highest $\mathrm{CPP}$-varies by each individual and by day (postinjury day 2 vs 3 ) following severe pediatric TBI. Furthermore, our hypothesis was that the optimal HOB position would be associated with improved or increased jugular vein outflow. Finally, we describe long-term follow-up survival outcomes.

\section{Methods \\ Data}

Pediatric patients were prospectively included in a single-center quaternary pediatric ICU between 2002 and 2008 . Inclusion criteria were age $<18$ years, no past medical history, accidental severe TBI with Glasgow Coma Scale (GCS) score $\leq 8$, the presence of a Camino intraparenchymal ICP monitoring device (Camino Laboratories), and no other neurosurgical procedure. The ICP monitor was zeroed prior to insertion. No patient had an external ventricular drain. Exclusion criteria included penetrating or abusive head trauma, GCS score of 3 with fixed and dilated pupils, and preexisting neurological, psychiatric, developmental disorder or other medical condition. The hospital's institutional review board approved the study, and parental informed consent was obtained.

\section{Measures}

Age and sex were recorded on admission to the pediatric ICU. Routine initial neurocritical care management included the following: supine position with the HOB elevated at $30^{\circ}$ and the hips extended, intubation and ventilation to normocarbia (arterial $\mathrm{CO}_{2} 35-39 \mathrm{~mm} \mathrm{Hg}$ ), adequate oxygenation (pulse oximetry saturation of $92 \%-$ 98\%), sedation/analgesia blockade with fentanyl and/or midazolam, neuromuscular blockade with vecuronium as needed, and phenylephrine or norepinephrine as needed to increase mean arterial pressure (MAP) to maintain minimum age-dependent CPP at 40-65 mm Hg. Our treatment protocol was consistent with the "Guidelines for the acute medical management of severe traumatic brain injury in infants, children, and adolescents."22

As part of pediatric neurocritical care management by our pediatric neurosurgical group, ICP was monitored by an intraparenchymal ICP device. Arterial blood pressure (ABP) was measured via a radial artery catheter and ABP was calibrated to the phlebostatic axis. $\mathrm{CPP}(\mathrm{mm} \mathrm{Hg})$ was calculated as the difference between mean ABP and ICP $(\mathrm{CPP}=\mathrm{MAP}-\mathrm{ICP})$. The degree of HOB elevation was routinely maintained at $30^{\circ}$ during the 1st postinjury day. From this baseline position, the degree of HOB elevation was varied between 6 positions: $0^{\circ}$ (head-flat or horizontal), $10^{\circ}, 20^{\circ}, 30^{\circ}, 40^{\circ}$, and $50^{\circ}$ on postinjury days 2 and 3 , as described by Rosner et al. ${ }^{17} \mathrm{~A}$ midline position of the head relative to the neck was maintained. If the patient's clinical status deteriorated (CPP $<40 \mathrm{~mm} \mathrm{Hg}$, concern for systemic hypertension and bradycardia with ICP $>25 \mathrm{~mm}$ $\mathrm{Hg} \geq 6$ minutes, systemic hypotension for age, or pulse oximetry oxygen saturation $<92 \%$ ), then that particular HOB angle was no longer used. Five minutes after the patient was placed in these 6 different head positions as described by Durward et al.." ${ }^{11}$ the patient's vital signs, ICP, and CPP were recorded every minute for a minimum of 6 minutes by a research assistant at the bedside. At the different head positions, duplex Doppler ultrasound (US) of the right and left IJVs was performed in single settings. The venous blood flow $\left(\mathrm{cm}^{3} / \mathrm{sec}\right)$ calculations were determined from the product of the measured cross-sectional area of the IJVs and the mean velocity of the vein, obtained at an insonation angle of approximately $60^{\circ}$, and measured at 30-second intervals or longer. Use of these variables should allow the US volumetric measurements to be sufficiently repeatable. Images were recorded by a registered US technologist and verified by a board-certified pediatric radiologist. This study was performed on 2 consecutive days-days 2 and 3 following injury-during the early afternoon (usually around $1 \mathrm{PM}$ ). When an $\mathrm{HOB}$ elevation that produced low ICP and high CPP was found, the patient was kept at that position for the rest of the day.

No patients had a central venous access device in their neck or upper extremity. Discharge and long-term Glasgow Outcome Scale-Extended (GOS-E) scores (up to 10 years following discharge) were obtained from available inpatient and outpatient charts. ${ }^{23-25}$

\section{Statistical Analysis}

Categorical variables are given as frequency and percentage, and categorical variables are presented as mean and SD or median and IQR, as appropriate. Analyses were stratified by days: postinjury day 2 and postinjury day 3 . To assess the overall difference in CPP and ICP between 
$30^{\circ}$ and other head positions (i.e., $0^{\circ}, 10^{\circ}, 20^{\circ}, 40^{\circ}$, and $50^{\circ}$ ) for all patients, a mixed-effects linear regression model was used to account for within-patient correlation generated from repeated measures in each patient by allowing for between-patient differences in CPP and ICP at baseline (i.e., at $30^{\circ} \mathrm{HOB}$ position for the current analysis). To assess differences in CPP and ICP at different head positions for each patient, the Kruskal-Wallis test was used as a global test of variance among the 6 different head positions, and the Wilcoxon rank-sum test was used for pairwise comparisons. The optimal head position for each day was defined when it demonstrated simultaneously the highest CPP and lowest ICP; CPP and ICP values for the optimal position for each day needed to be significantly different from all other position values. Patients without a single position or a set of positions that met the above optimal definition were coded as not having an optimal value.

The percent agreement was used to assess the absolute agreement in HOB positions with the highest CPP, the lowest ICP, or "optimal" between postinjury days 2 and 3 . Percent agreement and Cohen's kappa coefficient $(\kappa)$ were used to assess the agreement on whether $30^{\circ}$ was among the identified HOB positions that demonstrated the highest CPP, the lowest ICP, or optimal between postinjury days 2 and 3. Cohen's $\kappa$ is interpreted as: $\leq 0$ as indicating no agreement, $0.01-0.20$ as none to slight agreement, $0.21-0.40$ as fair agreement, $0.41-0.60$ as moderate agreement, $0.61-0.80$ as substantial agreement, and $0.81-1.00$ as almost perfect agreement. ${ }^{26}$

To explore whether the patients' demographic characteristics were associated with optimal HOB position, the patients were classified as those who demonstrated $30^{\circ}$ as one of the optimal HOB positions compared with those with an optimal $\mathrm{HOB}$ other than $30^{\circ}$ or no optimal HOB position on postinjury day 2 or 3 . The Wilcoxon rank-sum test was used to compare continuous variables, and the chi-square test or Fisher's exact test, as appropriate, was used to compare categorical variables between groups.

To explore the relationship between optimal head position and right and left IJV outflow, a mixed-effects linear regression model was used, allowing for baseline difference in venous outflow between patients. The difference in venous outflow between the right and left sides was also assessed using mixed-effects linear regression models. The distribution of studentized residuals and the Akaike information criterion were used for model diagnostics. A sensitivity analysis was conducted by adjusting for age, sex, and GCS score, dropping patients with no optimal position, or log-transforming the venous outflow values to ensure the robustness of the model and the results; sensitivity results were the same as above, thus not reported. All analyses were conducted using SAS software version 9.4 (SAS Institute).

\section{Results}

\section{Demographic and Clinical Characteristics}

Eighteen patients were analyzed in this study, and patient demographics are reported in Table 1. The mean age was 8.5 (SD 3.7) years, with the majority of the patients being male $(67 \%)$. All patients were involved in motor
TABLE 1. Patient demographics and CT findings

\begin{tabular}{|c|c|c|c|c|}
\hline $\begin{array}{l}\text { Case } \\
\text { No. }\end{array}$ & $\begin{array}{c}\text { Age } \\
\text { (yrs), } \\
\text { Sex }\end{array}$ & Lesion & Mechanism & $\begin{array}{c}\text { Initial } \\
\text { GCS } \\
\text { Score }\end{array}$ \\
\hline 1 & $6, M$ & DAI, SDH & MVA & 6 \\
\hline 2 & $7, \mathrm{M}$ & DAl & MVA & 8 \\
\hline 3 & $2, M$ & SAH, SDH & MVA & 3 \\
\hline 4 & $9, M$ & SAH, IPH & MVA vs pedestrian & 6 \\
\hline 5 & $10, F$ & Multiple IPH & MVA vs skateboard & 7 \\
\hline 6 & $11, \mathrm{~F}$ & DAI & MVA vs bicycle & 5 \\
\hline 7 & $14, \mathrm{~F}$ & Multiple IPH & MVA vs pedestrian & 3 \\
\hline 8 & $8, \mathrm{M}$ & $\mathrm{IVH}, \mathrm{SDH}$ & MVA vs pedestrian & 6 \\
\hline 9 & $8, M$ & Multiple IPH, SDH & MVA vs pedestrian & 5 \\
\hline 10 & $3, M$ & DAI, SDH & MVA & 6 \\
\hline 11 & $12, \mathrm{M}$ & IPH & MVA vs bicycle & 7 \\
\hline 12 & $2, M$ & DAI, IPH, IVH, SAH & MVA vs pedestrian & 5 \\
\hline 13 & $7, \mathrm{~F}$ & DAI, IPH & MVA vs pedestrian & 8 \\
\hline 14 & $13, \mathrm{M}$ & DAI, multiple IPH, SDH & MVA vs pedestrian & 3 \\
\hline 15 & $14, \mathrm{M}$ & $\mathrm{IPH}, \mathrm{SDH}$ & MVA & 7 \\
\hline 16 & $10, M$ & $\mathrm{IPH}, \mathrm{SDH}$ & MVA & 3 \\
\hline 17 & $7, F$ & $\mathrm{SAH}, \mathrm{SDH}$ & MVA vs pedestrian & 5 \\
\hline 18 & $10, F$ & IPH, SDH & MVA vs pedestrian & 8 \\
\hline
\end{tabular}

$\mathrm{DAI}=$ diffuse axonal injury; IPH = intraparenchymal hemorrhage; IVH = intraventricular hemorrhage; $\mathrm{MVA}=$ motor vehicle accident; $\mathrm{SAH}=$ subarachnoid hemorrhage; $\mathrm{SDH}=$ subdural hemorrhage.

vehicle accidents and had a median GCS score of 6. All patients in this study survived to hospital discharge. There were no instances of CPP $<40 \mathrm{~mm} \mathrm{Hg}$. While there were episodes of ICP $>25 \mathrm{~mm} \mathrm{Hg}$, all intracranial hypertension episodes responded to medical management, and there was no concern for herniation in any of the patients. Two episodes of systemic hypotension occurred with the HOB elevated to $50^{\circ}$ but only after being monitored at this angle for $>10$ minutes, which resolved by placing the HOB back to $30^{\circ}$. No episodes of desaturation were observed.

\section{Highest CPP}

On average, for all 18 patients $\mathrm{HOB}$ at $30^{\circ}$ did not demonstrate significantly higher CPP than all other HOB positions on postinjury day 2 , while on postinjury day 3 , HOB at $30^{\circ}$ was the position with the highest CPP (Supplementary Table 1).

Individually, CPP was significantly different on both postinjury days 2 and 3 across the different HOB positions (all $\mathrm{p}<0.001$, Supplementary Table 2). The HOB position with the highest CPP varied among the patients and days (Table 2, Fig. 1, and Supplementary Table 3). On postinjury day 2,9 patients had a single HOB position that demonstrated the highest CPP, 5 patients had more than $1 \mathrm{HOB}$ position that demonstrated statistically significant higher CPP than other positions, and 4 patients did not have any HOB position that demonstrated higher CPP. Six patients had $30^{\circ}$ as the HOB position with the highest CPP. On postinjury day 3,9 patients had a single HOB 
TABLE 2. Summary of HOB position with the highest CPP and lowest ICP on postinjury days 2 and 3

\begin{tabular}{|c|c|c|c|c|c|c|}
\hline \multirow[b]{3}{*}{ HOB Position $\left({ }^{\circ}\right)$} & \multicolumn{6}{|c|}{ No. of Patients (\%) } \\
\hline & \multicolumn{3}{|c|}{ Postinjury Day 2} & \multicolumn{3}{|c|}{ Postinjury Day 3} \\
\hline & Highest CPP & Lowest ICP & Optimal* & Highest CPP & Lowest ICP & Optimal ${ }^{*}$ \\
\hline 0 & $2(11.1)$ & $1(5.6)$ & $2(11.1)$ & $3(16.7)$ & $3(16.7)$ & $3(16.7)$ \\
\hline 10 & $1(5.6)$ & $2(11.1)$ & $2(11.1)$ & $1(5.6)$ & $1(5.6)$ & $2(11.1)$ \\
\hline 20 & $1(5.6)$ & $2(11.1)$ & $1(5.6)$ & $0(0)$ & $0(0)$ & $0(0)$ \\
\hline 30 & $2(11.1)$ & $3(16.7)$ & $3(16.7)$ & $4(22.2)$ & $4(22.2)$ & $4(22.2)$ \\
\hline 40 & $1(5.6)$ & $0(0)$ & $0(0)$ & $1(5.6)$ & $1(5.6)$ & $1(5.6)$ \\
\hline 50 & $2(11.1)$ & $2(11.1)$ & $2(11.1)$ & $0(0)$ & $1(5.6)$ & $1(5.6)$ \\
\hline Multiple positions & $5(27.8)$ & $5(27.8)$ & $3(16.7)$ & $6(33.3)$ & $6(33.3)$ & $2(11.1)$ \\
\hline No positions & $4(22.2)$ & $3(16.7)$ & $5(27.8)$ & $3(16.7)$ & $2(11.1)$ & $5(27.8)$ \\
\hline
\end{tabular}

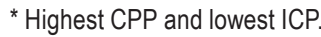

position that demonstrated the highest CPP, of which only 3 patients overlapped from postinjury day 2 . Six patients had more than $1 \mathrm{HOB}$ position that demonstrated higher CPP while 3 patients did not have any HOB position with higher CPP than other positions. Nine patients had $30^{\circ}$ as the HOB position with the highest CPP.

For 16 of the 18 patients (89\%), the HOB position that provided the highest CPP changed between postinjury days 2 and 3 , and there was only a fair agreement on whether $30^{\circ}$ was among the various HOB positions that demonstrated the highest CPP between postinjury days 2 and 3 (percent agreement: 61\% [11/18], Cohen's $\kappa=0.22$, $95 \%$ CI -0.20 to 0.65 ; Table 3 ).

\section{Lowest ICP}

On average, for all 18 patients $\mathrm{HOB}$ at $30^{\circ}$ did not demonstrate significantly lower ICP than all other HOB positions on either postinjury day 2 or 3 (Supplementary Table $1)$.

Individually, ICP was significantly different across the different HOB positions for each patient (all $p<0.03$, Supplementary Table 2). The HOB position with the lowest ICP varied among patients and days (Table 2, Fig. 1, and Supplementary Table 3). On postinjury day 2, 10 patients had a single HOB position that demonstrated the lowest ICP, 5 patients had more than $1 \mathrm{HOB}$ position with statistically significantly lower ICP than other positions, and 3 patients did not have any HOB position that demonstrated lower ICP than other positions. Seven patients had $30^{\circ}$ as the HOB position with the lowest ICP. On postinjury day 3,10 patients had a single $\mathrm{HOB}$ position that demonstrated the lowest ICP, 6 patients had more than 1 HOB position with statistically significantly lower ICP than other positions, and 2 patients did not have any HOB position that demonstrated lower ICP than other positions. Nine patients had $30^{\circ}$ as the HOB position with the lowest ICP.

For 17 of the 18 patients (94\%), the HOB that provided the lowest ICP changed between postinjury days 2 and 3 , and there was no agreement on whether $30^{\circ}$ was among the various HOB positions which demonstrated the lowest ICP between postinjury days 2 and 3 (percent agreement: $44 \%$ [8/18], Cohen's $\kappa=-0.11,95 \%$ CI -0.56 to 0.34; Table 3).

\section{Optimal HOB Position Per Patient for Each Day}

Table 2 provides a summary of the optimal HOB that demonstrated the highest CPP and lowest ICP for each patient on postinjury days 2 and 3 . For each day, there were 13 patients who had at least $1 \mathrm{HOB}$ position that simultaneously demonstrated the highest CPP and the lowest ICP (optimal HOB position), while 5 patients did not have one. Thirteen patients $(72 \%)$ had different optimal positions between the 2 study days. Overall, with 6 patients on each postinjury day, $30^{\circ}$ was the most frequent $\mathrm{HOB}$ position that simultaneously demonstrated the highest CPP and lowest ICP (Supplementary Table 3), but there was no agreement on whether $30^{\circ}$ was the optimal position for each patient between postinjury days 2 and 3 (percent agreement: 56\% [10/18], Cohen's $\kappa=0,95 \%$ CI -0.46 to 0.46 ; Table 3 ). Interestingly, $0^{\circ}$ or head-flat position in some patients ( 2 patients on postinjury day 2 and 4 patients on postinjury day 3 ) simultaneously demonstrated the highest CPP and lowest ICP (Supplementary Table 3).

Patients who had $30^{\circ}$ as the optimal HOB position on postinjury day 3 had a higher GCS score than those with an optimal HOB position other than $30^{\circ}(\mathrm{p}=0.0399)(\mathrm{Ta}-$ ble 4). However, the PILOT (Pediatric Intensity Level of Therapy) score was not different between the groups on postinjury day $2(\mathrm{p}=0.7710)$ or $3(\mathrm{p}=0.8084) .{ }^{27}$

\section{Optimal HOB Position and Venous Outflow}

Figure 2 demonstrates right and left IJV blood flows by each $\mathrm{HOB}$ position for each patient and highlights the optimal HOB position that was identified based on the lowest ICP and highest CPP. Within each patient, the optimal HOB position showed lower right IJV blood flow than the nonoptimal ones for both postinjury day $2(\mathrm{p}=0.0023)$ and postinjury day $3(\mathrm{p}=0.0033)$ (Table 5). On the contrary, there was no evidence that the optimal HOB positions and the nonoptimal ones were different in the left IJV blood flow (Table 3). Comparing the right and left IJV blood flows within each patient, the IJV flow was lower on the left side than the on the right side on both postinjury day $2(\mathrm{p}<0.0001)$ and postinjury day $3(\mathrm{p}<0.0001$, Fig. 2 , Table 6). Sensitivity analysis adjusted for age, sex, and GCS score, excluding patients without optimal position, 


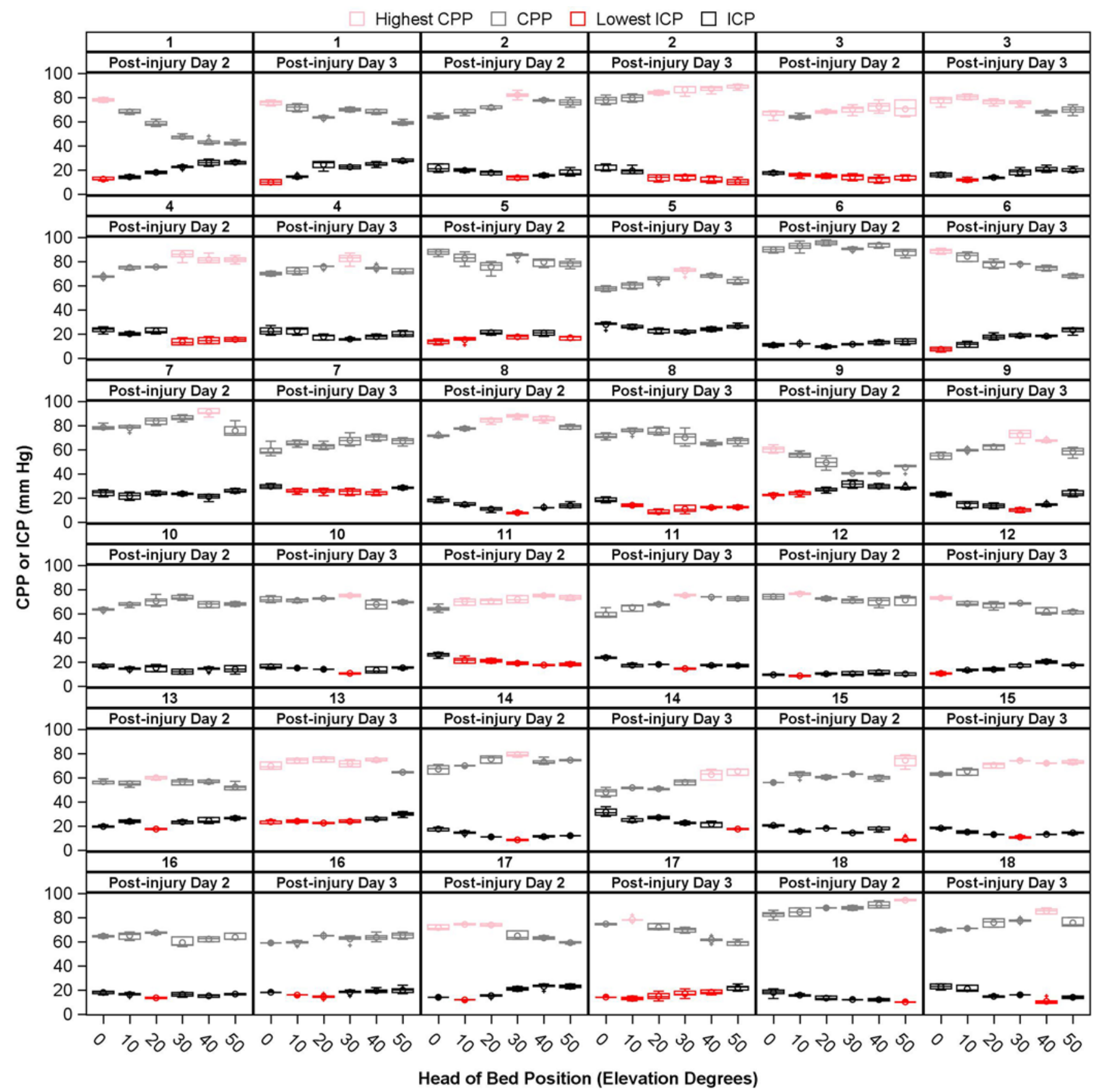

FIG. 1. Box plots showing the CPP and ICP values at each HOB for each patient on postinjury days 2 and 3. Positions with highest CPP or lowest ICP are shown in red. The horizontal line in the box represents the median, the lower and upper borders of the box are 25th and 75th percentile. The circle represents the mean, the lower and upper whiskers are the minimum and maximum values, respectively, within the lower and upper fence (i.e., 1.5 $\times$ IQR below the 25th percentile and 1.5 $\times$ IQR above 75th percentile). The plus symbols are outliers (defined as values out of the lower and upper fences). Figure is available in color online only.

or using log-transformed blood flow values, demonstrated consistent results.

\section{Long-Term Outcome}

Thirteen of the 18 patients $(72 \%)$ were transferred to inpatient rehabilitation. The remaining 5 patients $(28 \%)$ who were discharged home underwent outpatient rehabili- tation follow-up. The patients were followed up using the GOS-E for up to 10 years after discharge: 1 patient (6\%) was lost to follow-up after discharge and $3(17 \%)$ were followed up to 1 year, 3 (17\%) up to 5 years, and $11(61 \%)$ up to 10 years. As demonstrated in Fig. 3, while most of the patients functionally improved with time, the youngest children $(<4$ years of age) had the worst outcomes at 
TABLE 3. Contingency tables between postinjury days 2 and 3

\begin{tabular}{|c|c|c|c|c|c|}
\hline \multirow[t]{2}{*}{ Position } & \multirow[t]{2}{*}{ Postinjury Day 2} & \multicolumn{2}{|c|}{ Postinjury Day 3} & \multirow[t]{2}{*}{ Cohen's $\kappa(95 \% \mathrm{Cl})$} & \multirow[t]{2}{*}{$\%$ Agreement } \\
\hline & & No & Yes & & \\
\hline \multirow{2}{*}{$30^{\circ}$ was optimal position } & No & 8 & 4 & \multirow{2}{*}{$0(-0.46$ to 0.46$)$} & \multirow{2}{*}{$56 \%(10 / 18)$} \\
\hline & Yes & 4 & 2 & & \\
\hline \multirow{3}{*}{$30^{\circ}$ was position w/ highest CPP } & & No & Yes & \multirow{3}{*}{$0.22(-0.20$ to 0.65$)$} & \multirow{3}{*}{$61 \%(11 / 18)$} \\
\hline & No & 7 & 5 & & \\
\hline & Yes & 2 & 4 & & \\
\hline \multirow{3}{*}{$30^{\circ}$ was position $\mathrm{w} /$ lowest ICP } & & No & Yes & \multirow{3}{*}{$-0.11(-0.56$ to 0.34$)$} & \multirow{3}{*}{$44 \%(8 / 18)$} \\
\hline & No & 5 & 6 & & \\
\hline & Yes & 4 & 3 & & \\
\hline
\end{tabular}

10 years postinjury and demonstrated severe to moderate disability.

\section{Discussion}

In this prospective study of 18 children with severe pediatric TBI, the various HOB positions overall resulted in different CPP and ICP on both postinjury days 2 and 3. In- dividually, many of the patients had a single HOB position that demonstrated the highest CPP or the lowest ICP. However, some of the patients had more than $1 \mathrm{HOB}$ position that resulted in the highest CPP or the lowest ICP, whereas others did not have any positions that provided the highest $\mathrm{CPP}$ or the lowest ICP. For most of the patients, the HOB position that provided the highest CPP or the lowest ICP changed on postinjury days 2 and 3 . While the majority

TABLE 4. Association of patients' characteristics and HOB positions

\begin{tabular}{|c|c|c|c|c|c|c|c|}
\hline \multirow[b]{3}{*}{ Variable } & \multirow[b]{3}{*}{ All Patients } & \multicolumn{3}{|c|}{ Postinjury Day 2} & \multicolumn{3}{|c|}{ Postinjury Day 3} \\
\hline & & \multicolumn{2}{|c|}{$30^{\circ}$ Was Optimal Position } & \multirow{2}{*}{$\begin{array}{c}p \\
\text { Value }\end{array}$} & \multicolumn{2}{|c|}{$30^{\circ}$ Was Optimal Position } & \multirow{2}{*}{$\begin{array}{c}p \\
\text { Value }\end{array}$} \\
\hline & & No & Yes & & No & Yes & \\
\hline No. of patients & 18 & $12(67)$ & $6(33)$ & & $12(67)$ & $6(33)$ & \\
\hline Median age, yrs & $8.5(7-11)$ & $9(6.5-10.5)$ & $8.5(7-12)$ & $>0.99$ & $9.5(6.5-10.5)$ & $7.5(7-12)$ & 0.9624 \\
\hline Male sex & $12(66.7)$ & $6(50)$ & $6(100)$ & 0.0537 & $7(58.3)$ & $5(83.3)$ & 0.6000 \\
\hline Median initial GCS score & $6(5-7)$ & $5.5(5-7)$ & $6(3-7)$ & $>0.99$ & $5(3-6)$ & $7(6-8)$ & 0.0399 \\
\hline \multicolumn{8}{|l|}{ Type of lesion } \\
\hline DAl & $7(38.9)$ & $5(41.7)$ & $2(33.3)$ & $>0.99$ & $4(33.3)$ & $3(50)$ & 0.6267 \\
\hline $\mathrm{SDH}$ & $10(55.6)$ & $7(58.3)$ & $3(50)$ & $>0.99$ & $7(58.3)$ & $3(50)$ & $>0.99$ \\
\hline $\mathrm{SAH}$ & $4(22.2)$ & $2(16.7)$ & $2(33.3)$ & 0.5686 & $4(33.3)$ & $0(0)$ & 0.2451 \\
\hline $\mathrm{IVH}$ & $2(11.1)$ & $1(8.3)$ & $1(16.7)$ & $>0.99$ & $2(16.7)$ & $0(0)$ & 0.5294 \\
\hline $\mathrm{IPH}$ & $7(38.9)$ & $5(41.7)$ & $2(33.3)$ & $>0.99$ & $4(33.3)$ & $3(50)$ & 0.6267 \\
\hline Multiple IPH & $4(22.2)$ & $3(25)$ & $1(16.7)$ & $>0.99$ & $3(25)$ & $1(16.7)$ & $>0.99$ \\
\hline Mechanism & & & & $>0.99$ & & & 0.5905 \\
\hline MVA & $6(33.3)$ & $4(33.3)$ & $2(33.3)$ & & $3(25)$ & $3(50)$ & \\
\hline MVA vs bicycle & $2(11.1)$ & $1(8.3)$ & $1(16.7)$ & & $1(8.3)$ & $1(16.7)$ & \\
\hline MVA vs pedestrian & $9(50)$ & $6(50)$ & $3(50)$ & & $7(58.3)$ & $2(33.3)$ & \\
\hline MVA vs skateboard & $1(5.6)$ & $1(8.3)$ & $0(0)$ & & $1(8.3)$ & $0(0)$ & \\
\hline Median PILOT score & $4(2-7)$ & $5.5(2-7)$ & $4(4-4)$ & 0.7710 & $4(2-7)$ & $4(4-7)$ & 0.8084 \\
\hline \multicolumn{8}{|l|}{ Type of therapy } \\
\hline Neuromuscular blockade & $13(72.2)$ & $8(67.7)$ & $5(83.3)$ & 0.6148 & $8(66.7)$ & $5(83.3)$ & 0.6148 \\
\hline Mannitol & $3(16.7)$ & $2(16.7)$ & $1(16.7)$ & $>0.99$ & $2(16.7)$ & $1(16.7)$ & $>0.99$ \\
\hline $3 \%$ saline & $7(38.9)$ & $6(50)$ & $1(16.7)$ & 0.3156 & $5(41.7)$ & $2(33.3)$ & $>0.99$ \\
\hline Vasopressor & $1(5.6)$ & $1(8.3)$ & $0(0)$ & $>0.99$ & $0(0)$ & $1(16.7)$ & 0.3333 \\
\hline
\end{tabular}

PILOT $=$ Pediatric Intensity Level of Therapy.

Values are presented as the number of patients (\%) or median (IQR).

All patients in the study received sedation (narcotics and benzodiazepines) and ventilation (normocarbia). 


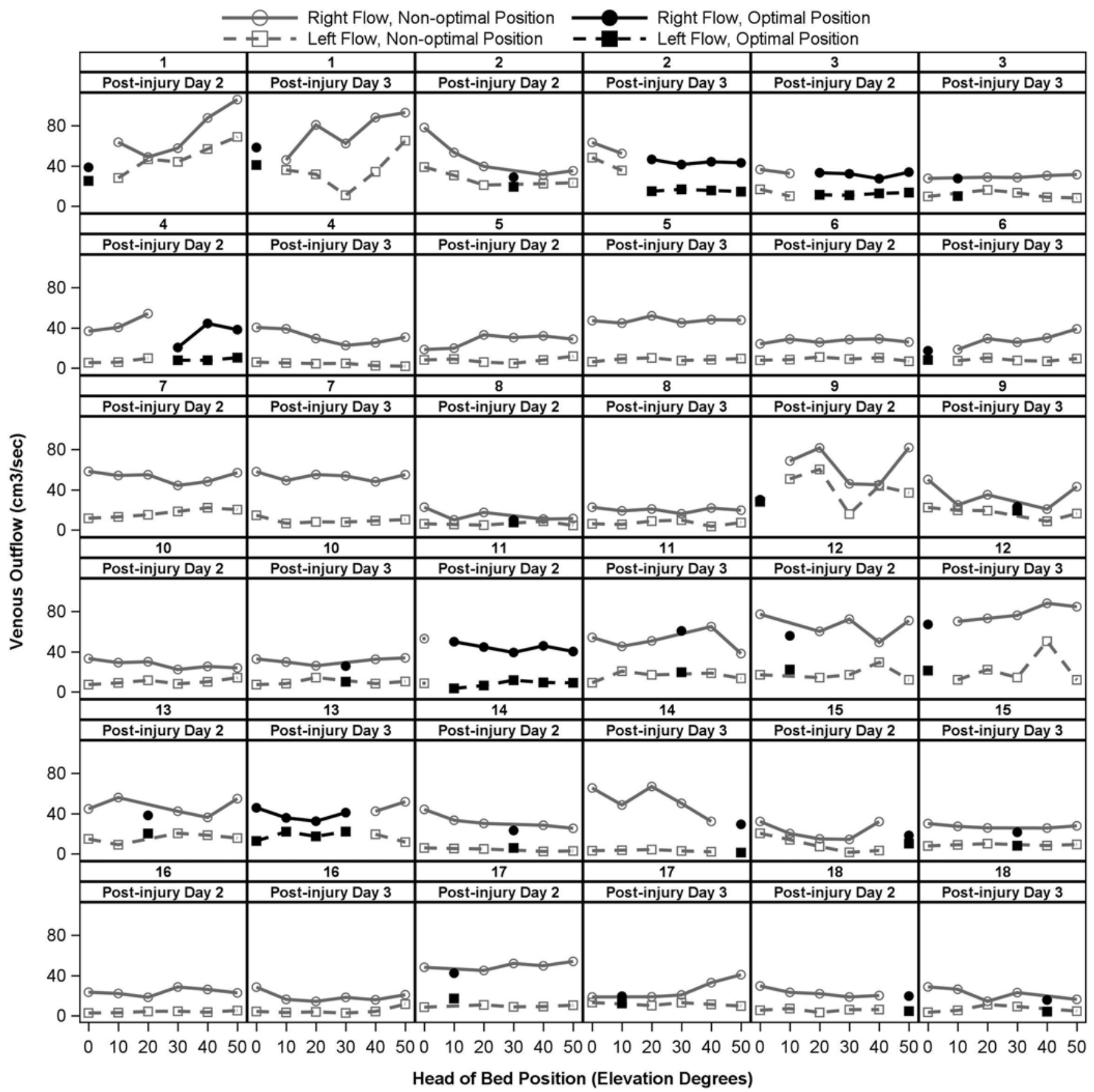

FIG. 2. Right and left IJV blood flows by optimal and nonoptimal HOB positions on postinjury days 2 and 3 .

of the patients had at least $1 \mathrm{HOB}$ position that provided the optimal HOB position (the position that simultaneously demonstrated the highest CPP and the lowest ICP), some patients did not have an optimal HOB position. Collectively, our study suggests that the HOB position that minimizes ICP and/or promotes the highest CPP may vary by each individual and by each day postinjury following severe pediatric TBI.

Most of the previous studies on HOB position have been in the adult population with severe TBI. Feldman et al. studied 22 adult patients and analyzed ICP, CPP, and cerebral blood flow $(\mathrm{CBF})$ with $\mathrm{HOB}$ position at $30^{\circ}$ vs $0^{\circ}$ (head-flat or horizontal position). They found that raising the $\mathrm{HOB}$ to $30^{\circ}$ was very effective in lowering ICP with no effect on CPP and CBF. ${ }^{12}$ While our current pediatric study demonstrated that for an individual patient on a given day after injury, the HOB position that best lowers ICP and/or improves the CPP is not always $30^{\circ}$; it is interesting that our study did show that $30^{\circ}$ was the most frequent position associated with the lowest ICP and/or the highest CPP values on both days postinjury. In contrast, Rosner and Coley argued that keeping adult patients at $0^{\circ}$ (head- 
TABLE 5. Association of optimal HOB position and IJV blood flow

\begin{tabular}{|c|c|c|c|c|c|c|c|c|}
\hline \multirow[b]{3}{*}{ Variable } & \multicolumn{4}{|c|}{ Postinjury Day 2} & \multicolumn{4}{|c|}{ Postinjury Day 3} \\
\hline & \multicolumn{2}{|c|}{ Rt IJV Flow, $\mathrm{cm}^{3} / \mathrm{sec}$} & \multicolumn{2}{|c|}{ Lt IJV Flow, $\mathrm{cm}^{3} / \mathrm{sec}$} & \multicolumn{2}{|c|}{ Rt IJV Flow, $\mathrm{cm}^{3} / \mathrm{sec}$} & \multicolumn{2}{|c|}{ Lt IJV Flow, $\mathrm{cm}^{3} / \mathrm{sec}$} \\
\hline & Estimate (SE) & $p$ Value & Estimate (SE) & $p$ Value & Estimate (SE) & p Value & Estimate (SE) & $p$ Value \\
\hline \multicolumn{9}{|l|}{ Fixed effects } \\
\hline Intercept & $40.7(3.9)$ & $<0.0001$ & $14.6(2.8)$ & $<0.0001$ & $40.4(4.2)$ & $<0.0001$ & $12.8(2.1)$ & $<0.0001$ \\
\hline Optimal vs nonoptimal position & $-10.9(2.8)$ & 0.0023 & $-1.8(1.9)$ & 0.3503 & $-8.5(2.3)$ & 0.0033 & $-1.6(2.0)$ & 0.4376 \\
\hline \multicolumn{9}{|l|}{ Covariance parameter } \\
\hline $\mathrm{U}(1,1)$ & $247.7(90.9)$ & 0.0032 & $127.4(46.2)$ & 0.0029 & $296.3(105.5)$ & 0.0025 & $67.4(26.0)$ & 0.0048 \\
\hline Residual & $100.1(15.0)$ & $<0.0001$ & $42.8(6.4)$ & $<0.0001$ & $65.4(9.8)$ & $<0.0001$ & $46.9(7.0)$ & $<0.0001$ \\
\hline \multicolumn{9}{|l|}{ Model fit statistics } \\
\hline AIC (smaller is better) & 847.2 & & 760.1 & & 811.8 & & 758.4 & \\
\hline
\end{tabular}

$\mathrm{AIC}=$ Akaike information criterion.

There were 108 (18 patients $\times 6$ positions) observations on each side and day.

flat or horizontal position) provided the maximal CPP. ${ }^{17}$ They evaluated 18 adults with intracranial hypertension and measured ICP and CPP with their $\mathrm{HOB}$ elevated from $0^{\circ}$ to $50^{\circ}$ in $10^{\circ}$ increments. Over the course of the study, they found that with every $10^{\circ}$ of head elevation, on average the ICP decreased by $1 \mathrm{~mm} \mathrm{Hg}$ and CPP decreased by $2-3 \mathrm{~mm} \mathrm{Hg}$. No increase in CPP was found with any elevation of head position, and maximal CPP was always demonstrated at the head-flat or horizontal position, causing Rosner and Coley to claim that the optimum position for increased $\mathrm{CBF}$ and $\mathrm{CPP}$ was in the horizontal position. ${ }^{17}$ Similar to Rosner and Coley's study, we did find in our pediatric study that, while not the majority, individual patients on individual days did experience the highest CPP with the head positioned at $0^{\circ}$. Ropper et al. evaluated ICP in 19 adults at $60^{\circ}$ and $0^{\circ}$. Ten patients had lower ICP with the $\mathrm{HOB}$ at $60^{\circ}, 2$ patients had lower ICP at $0^{\circ}$, and 7 patients were unchanged. No data on CPP were reported..$^{16}$ As can be seen in our current pediatric study, we had a minority of individual patients whose HOB position changes had no effect on CPP and/or ICP.

The only previous pediatric study was conducted by Agbeko et al., who evaluated ICP and CPP in relation to HOB positions that ranged from $0^{\circ}$ to $40^{\circ}$ elevation (in $10^{\circ}$ increments) in 8 pediatric patients with severe TBI..$^{21}$ They reported that there was a negative and linear relationship between the change in HOB position and ICP, with the lowest ICP usually, but not always, achieved by the highest HOB position, whereas the CPP was unaffected. This differs from our pediatric study, as we observed a subset of patients who had the lowest ICP and the highest CPP with a head-flat or horizontal bed position $\left(0^{\circ}\right)$. Our study also differs from that of Agbeko et al. as we analyzed more patients and up to $50^{\circ}$ of elevation. Furthermore, to the best of our knowledge, our current study is the only study that analyzed HOB positions on 2 consecutive days for each patient following severe TBI. Collectively, the aforementioned studies suggest that the pathophysiological relationship between HOB position and CPP and ICP is clearly quite complex.

As it is thought that optimizing the head position lowers ICP via hydrostatic displacement of CSF from the cranial vault to the spinal subarachnoid space and increasing venous drainage from the brain, ${ }^{13}$ we hypothesized that an optimal HOB position would increase IJV outflow. The IJVs are commonly found to be asymmetrical, and many studies quote $68 \%-80 \%$ dominance of the right IJV outflow. ${ }^{28-31}$ In accordance with this, we found that the right IJV blood flow was higher than that in the left. However, contrary to our hypothesis was the fact that the right IJV blood flow was actually less at an individual patient's optimal HOB position (the position that provided the low-

TABLE 6. Right and left IJV blood flow by postinjury day

\begin{tabular}{|c|c|c|c|c|}
\hline \multirow[b]{2}{*}{ Variable } & \multicolumn{2}{|c|}{ Postinjury Day 2} & \multicolumn{2}{|c|}{ Posinjury Day 3} \\
\hline & Estimate (SE) & p Value & Estimate (SE) & $p$ Value \\
\hline \multicolumn{5}{|l|}{ Fixed effects } \\
\hline Intercept & $38.4(3.1)$ & $<0.0001$ & $38.9(2.9)$ & $<0.0001$ \\
\hline Lt vs rt IJV flow, $\mathrm{cm}^{3} / \mathrm{sec}$ & $-24.2(1.4)$ & $<0.0001$ & $-26.4(1.4)$ & $<0.0001$ \\
\hline \multicolumn{5}{|l|}{ Covariate parameter } \\
\hline$U(1,1)$ & $157.7(56.9)$ & 0.0028 & $133.5(48.6)$ & 0.003 \\
\hline Residual & $98.9(10.0)$ & $<0.0001$ & $98.8(10.0)$ & $<0.0001$ \\
\hline \multicolumn{5}{|l|}{ Model fitness statistics } \\
\hline $\mathrm{AIC}$ & 1654.9 & & 1651.9 & \\
\hline
\end{tabular}

There were 216 observations ( 18 patients $\times 6$ positions $\times 2$ sides). 


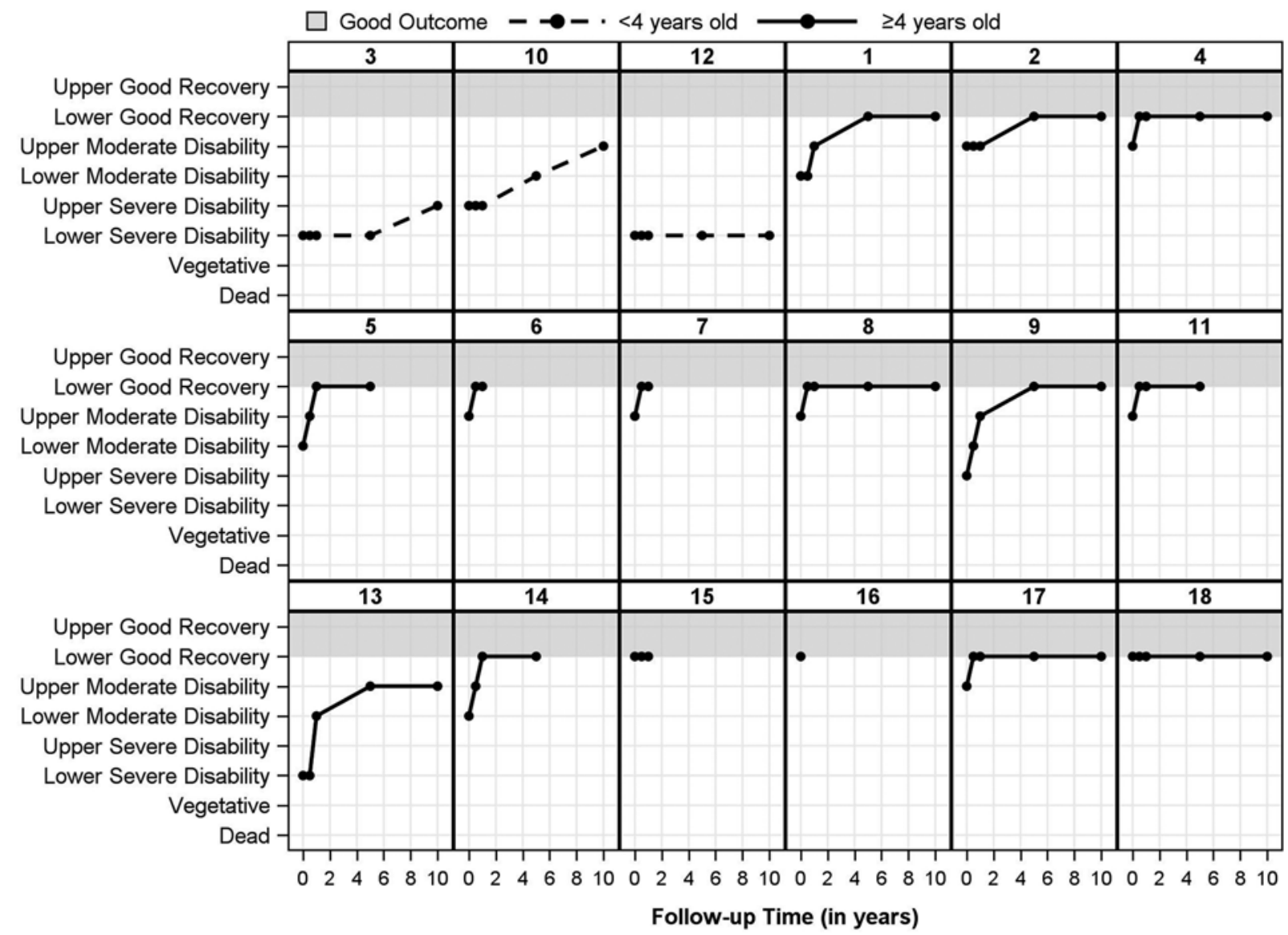

FIG. 3. GOS-E score by patient: long-term posthospital discharge survival outcomes.

est ICP and the highest CPP) compared with the same individual's nonoptimal position on both postinjury days. While the mechanism for this apparent paradoxical result is unclear, one possibility is that at the optimal HOB position, the intracranial venous compliance improved, which then allowed an increase in cerebral venous blood volume with a subsequent decrease in IJV outflow. While further studies are clearly needed, the changes in venous compliance may affect the IJV diameter and the local blood flow velocity. Other studies to correlate the IJV blood flow may involve CBF either invasively with a CBF monitor or noninvasively via transcranial Doppler monitoring.

Lastly, we described the long-term outcomes of our 18 patients, who all survived. Most of our patients were transferred to inpatient rehabilitation facilities, while the rest were discharged to home with outpatient therapy. Most of the patients had functional improvement, but patients who were younger than 4 years at the time of injury had the worst functional long-term outcomes. This is consistent with previous literature demonstrating poorer outcome in the youngest pediatric population following severe TBI, despite the fact that the younger age group has increased levels of plasticity. ${ }^{32-35}$

\section{Limitations}

Limitations to our study include the fact that we exclud- ed pediatric patients with TBI due to abuse, which is one of the leading causes of severe TBI in the youngest population. We also excluded patients with other comorbidities, such as those with polytrauma. None of the patients in this study needed emergency neurosurgical evacuation of hematomas or decompressive craniotomy, which may contribute to and confound changes in ICP and CPP. It is possible that varying the head position by $10^{\circ}$ can influence the subsequent ICP measurements; our study was performed in the early afternoon, so we cannot determine whether there may be variability throughout a single day. Furthermore, our follow-up was to demonstrate that the patients safely tolerated the study and survived. Moreover, with a limited number of patients, the results of the exploratory analysis of the association between patients' characteristics and optimal HOB position should be interpreted with caution. Future studies should address the role of optimal HOB position and outcome.

\section{Conclusions}

Our data demonstrate that the optimal HOB position needs to be addressed for each pediatric patient individually and even on a daily basis. Future studies should incorporate individualized responses to head positioning on a more frequent basis (such as continual hourly basis if safely tolerated) while measuring ICP, CPP, and other neu- 
romonitoring parameters, such as brain tissue oxygenation and $\mathrm{CBF}$, with the ultimate hope of improving outcome.

\section{Acknowledgments}

This investigation was supported (in part) by the Public Health Services Research Grant M01-RR00240 from the National Institutes of Health (J.W.H.).

\section{References}

1. Araki T, Yokota H, Morita A. Pediatric traumatic brain injury: characteristic features, diagnosis, and management. Neurol Med Chir (Tokyo). 2017;57(2):82-93.

2. Dewan MC, Mummareddy N, Wellons JC III, Bonfield CM. Epidemiology of global pediatric traumatic brain injury: qualitative review. World Neurosurg. 2016;91:497-509.

3. Taylor CA, Bell JM, Breiding MJ, Xu L. Traumatic brain injury-related emergency department visits, hospitalizations, and deaths-United States, 2007 and 2013. MMWR Surveill Summ. 2017;66(9):1-16.

4. Thurman DJ. The epidemiology of traumatic brain injury in children and youths: a review of research since 1990. J Child Neurol. 2016;31(1):20-27.

5. Kochanek PM, Tasker RC, Carney N, et al. Guidelines for the management of pediatric severe traumatic brain injury, third edition: update of the Brain Trauma Foundation guidelines, executive summary. Pediatr Crit Care Med. 2019;20(3): 280-289.

6. Bruce DA, Berman WA, Schut L. Cerebrospinal fluid pressure monitoring in children: physiology, pathology and clinical usefulness. Adv Pediatr. 1977;24:233-290.

7. Lipe HP, Mitchell PH. Positioning the patient with intracranial hypertension: how turning and head rotation affect the internal jugular vein. Heart Lung. 1980;9(6):1031-1037.

8. Lovell AT, Marshall AC, Elwell CE, et al. Changes in cerebral blood volume with changes in position in awake and anesthetized subjects. Anesth Analg. 2000;90(2):372-376.

9. Magnaes B. Body position and cerebrospinal fluid pressure. Part 1: clinical studies on the effect of rapid postural changes. J Neurosurg. 1976;44(6):687-697.

10. Shapiro HM. Intracranial hypertension: therapeutic and anesthetic considerations. Anesthesiology. 1975;43(4):445-471.

11. Durward QJ, Amacher AL, Del Maestro RF, Sibbald WJ. Cerebral and cardiovascular responses to changes in head elevation in patients with intracranial hypertension. $J$ Neurosurg. 1983;59(6):938-944.

12. Feldman Z, Kanter MJ, Robertson CS, et al. Effect of head elevation on intracranial pressure, cerebral perfusion pressure, and cerebral blood flow in head-injured patients. J Neurosurg. 1992;76(2):207-211.

13. Kenning JA, Toutant SM, Saunders RL. Upright patient positioning in the management of intracranial hypertension. Surg Neurol. 1981;15(2):148-152.

14. Meixensberger J, Baunach S, Amschler J, et al. Influence of body position on tissue-pO2, cerebral perfusion pressure and intracranial pressure in patients with acute brain injury. $\mathrm{Neu}$ rol Res. 1997;19(3):249-253.

15. Ng I, Lim J, Wong HB. Effects of head posture on cerebral hemodynamics: its influences on intracranial pressure, cerebral perfusion pressure, and cerebral oxygenation. Neurosurgery. 2004;54(3):593-598.

16. Ropper AH, O'Rourke D, Kennedy SK. Head position, intracranial pressure, and compliance. Neurology. 1982;32(11): $1288-1291$.

17. Rosner MJ, Coley IB. Cerebral perfusion pressure, intracranial pressure, and head elevation. J Neurosurg. 1986;65(5): 636-641.

18. Schulz-Stübner S, Thiex R. Raising the head-of-bed by 30 degrees reduces ICP and improves CPP without compromising cardiac output in euvolemic patients with traumatic brain injury and subarachnoid haemorrhage: a practice audit. Eur $J$ Anaesthesiol. 2006;23(2):177-180.

19. Winkelman C. Effect of backrest position on intracranial and cerebral perfusion pressures in traumatically brain-injured adults. Am J Crit Care. 2000;9(6):373-382.

20. Woischneck D, Gaab MR. Intracranial pressure with head elevation. J Neurosurg. 1992;77(4):651-652.

21. Agbeko RS, Pearson S, Peters MJ, et al. Intracranial pressure and cerebral perfusion pressure responses to head elevation changes in pediatric traumatic brain injury. Pediatr Crit Care Med. 2012;13(1):e39-e47.

22. Adelson PD, Bratton SL, Carney NA, et al. Guidelines for the acute medical management of severe traumatic brain injury in infants, children, and adolescents. Chapter 17. Critical pathway for the treatment of established intracranial hypertension in pediatric traumatic brain injury. Pediatr Crit Care Med. 2003;4(3)(suppl):S65-S67.

23. Beers SR, Wisniewski SR, Garcia-Filion P, et al. Validity of a pediatric version of the Glasgow Outcome Scale-Extended. $J$ Neurotrauma. 2012;29(6):1126-1139.

24. Jennett B, Snoek J, Bond MR, Brooks N. Disability after severe head injury: observations on the use of the Glasgow Outcome Scale. J Neurol Neurosurg Psychiatry. 1981;44(4): 285-293.

25. Teasdale GM, Pettigrew LE, Wilson JT, et al. Analyzing outcome of treatment of severe head injury: a review and update on advancing the use of the Glasgow Outcome Scale. J Neurotrauma. 1998;15(8):587-597.

26. Landis JR, Koch GG. The measurement of observer agreement for categorical data. Biometrics. 1977;33(1):159-174.

27. Shore PM, Hand LL, Roy L, et al. Reliability and validity of the Pediatric Intensity Level of Therapy (PILOT) scale: a measure of the use of intracranial pressure-directed therapies. Crit Care Med. 2006;34(7):1981-1987.

28. Bos MJ, van Loon RF, Heywood L, et al. Comparison of the diameter, cross-sectional area, and position of the left and right internal jugular vein and carotid artery in adults using ultrasound. J Clin Anesth. 2016;32:65-69.

29. Cormio M, Robertson CS. Ultrasound is a reliable method for determining jugular bulb dominance. $J$ Neurosurg Anesthesiol. 2001;13(3):250-254.

30. Czyżewska D, Krysiuk K, Dobrzycki K, Ustymowicz A. Ultrasound assessment of the jugular and vertebral veins in healthy individuals: selected physiological aspects and morphological parameters. J Ultrason. 2015;15(62):267-273.

31. Czyzewska D, Ustymowicz A, Kosel J. Internal jugular veins must be measured before catheterization. J Clin Anesth. 2015;27(2):129-131.

32. Anderson V, Catroppa C, Morse S, et al. Functional plasticity or vulnerability after early brain injury? Pediatrics. 2005; 116(6):1374-1382.

33. Anderson VA, Catroppa C, Haritou F, et al. Identifying factors contributing to child and family outcome 30 months after traumatic brain injury in children. J Neurol Neurosurg Psychiatry. 2005;76(3):401-408.

34. Anderson VA, Morse SA, Catroppa C, et al. Thirty month outcome from early childhood head injury: a prospective analysis of neurobehavioural recovery. Brain. 2004;127(Pt 12):2608-2620.

35. Ewing-Cobbs L, Prasad MR, Kramer L, et al. Late intellectual and academic outcomes following traumatic brain injury sustained during early childhood. J Neurosurg. 2006;105(4) (suppl):287-296.

\section{Disclosures}

The authors report no conflict of interest concerning the materi- 
als or methods used in this study or the findings specified in this paper.

\section{Author Contributions}

Conception and design: Huh. Acquisition of data: Leavesley, Bellah, Huh. Analysis and interpretation of data: Lang, Bellah, Kim, Huh. Drafting the article: Lang, Valeri, Huh. Critically revising the article: Lang, Valeri, Zhang, Storm, Heuer, Bellah, Kim, Griffis, Kilbaugh, Huh. Reviewed submitted version of manuscript: all authors. Approved the final version of the manuscript on behalf of all authors: Lang. Statistical analysis: Zhang, Griffis. Administrative/technical/material support: Lang. Study supervision: Lang, Huh.

\section{Supplemental Information}

Online-Only Content

Supplemental material is available with the online version of the article.

Supplementary Tables 1-3. https://thejns.org/doi/suppl/10. 3171/2020.4.PEDS20102.

\section{Correspondence}

Shih-Shan Lang: Children's Hospital of Philadelphia, PA. shihshan.lang@uphs.upenn.edu. 